\title{
Dietary fatty acids and colon cancer
}

\author{
Elizabeth K. Lund
}

Institute of Food Research, Norwich, UK

Abstract

Although epidemiological evidence to support a preventive role for $n-3$ fatty acids in relation to colorectal cancer development is currently not convincing, there is a substantial and consistent literature based on a diverse range of animal models and in vitro studies to suggest that an increase in the ratio of n-3 to n- 6 fatty acids in the diet is likely to be beneficial in this respect. Studies using intermediate markers of disease risk are strongly indicative of a protective effect of $n-3$ fatty acid consumption. The epidemiological evidence suffers from a lack of intervention studies with tumour as an endpoint, and in many of the observational studies, a low level of consumption or small ranges of intake combined with the fact that most studies were not designed specifically to identify dietary fat quality. A wide range of potential mechanisms has been investigated in relation to how different polyunsaturated fatty acids might modify risk, including alterations in luminal, paracrine and endocrine factors, as well as direct effects on cell division, differentiation and apoptosis.

Keywords: $n$-3 fatty acids; colorectal cancer; nuclear receptors; prostaglandins inflammation

Abbreviations: EGF: epidermal growth factor; EPA: eicosapentaenoic acid; DHA: docosahexaenic acid; DMH: dimethylhydrazine; IGF: insulin-like growth factor; IGFBP: IGF binding protein; IL: interleukin; PGE: prostaglandin; PPAR: peroxisome proliferator activated receptor; PUFA: polyunsaturated fatty acid; RxR: retinoid $X$ receptor; $S N P$ : single nucleotide polymorphism.

\section{Introduction}

$\mathrm{C}$ olorectal cancer is the second or third most prevalent cancer in the majority of Westernized countries. In Japan the incidence has increased more than four-fold since the 1960s, with the number of cases reported each year per 100000 now exceeding that of the UK $(1,2)$. Likewise, second generation Japanese people living in the USA have similar colorectal cancer rates to those of the general population. These data suggest that the vast majority of cases of colorectal cancer are of an environmental origin and therefore should be preventable (2). It has been estimated that in the region of $60-80 \%$ of cases may be attributable to a dietary cause, and it is well recognized that high seafood consumption, and the associated high ratio of $n$ 3:n-6 fatty acids in the diet, is one characteristic of the Japanese lifestyle that may be lost as a population moves towards a more Westernized way of life.

\section{Aetiology of colorectal cancer}

Colorectal cancer develops over many years as a result of an accumulation of epigenetic and genetic changes that result in a loss of control of cell proliferation and a failure of damaged cells to undergo apoptosis (3). Over a period of decades normal healthy mucosa develops small areas of focal damage termed aberrant crypt foci, with small adenomas detectable on the luminal surface. A small percentage of these preneoplastic lesions will develop into tumours, which may eventually metastasize. The healthy colon is lined by a simple columnar epithelium which has a three-dimensional structure consisting of numerous invaginations to form secretory glands or crypts (4). At the base of the crypt there is a stem-cell compartment from which two main cell lineages develop: absorptive epithelial cells and exocrine secretory cells or goblet cells. In the healthy tissue stem cells are very sensitive to apoptosis in response to any damaging agent, thereby minimizing clonal expansion of mutated cells. By far the most prevalent cell type is the absorptive columnar cell. Above the stem-cell zone there is a region of the crypt that contains proliferative cells that move up the crypt over a period of 3-4 days. As cells reach a point about half way up the crypt they stop dividing and differentiate. The apparently normal mucosa of tumour patients is characterized by a failure of the epithelial cells to stop dividing at an appropriate point and thus create an expanded proliferative zone. Numerous experiments have shown the potential for a 
range of dietary factors to normalize this process as well as to increase apoptosis and thus facilitate the removal of damaged cells. Underlying this surface layer of cells, within the lamina propria, there are both fibroblasts and a diverse range of different inflammatory cells, which in places form into localized areas of specialized lymphoid tissue equivalent to, but rather smaller than, the Peyer's patches found in the small intestine (5). While the vast majority of colonic tumours develop from the epithelial population, the presence of both lamina propria and intraepithelial lymphocytes has an impact on colonic health, as illustrated by the increase in crypt-cell proliferation associated with chronic inflammatory bowel diseases such as ulcerative colitis and Crohn's disease, as well as in some cases of irritable bowel syndrome, where inflammation is now recognized to play a part.

The role of paracrine signalling molecules produced by T-cells, dendritic cells and macrophages present in the colon, as well as through direct contact between such cells in mediating changes in epithelial cell proliferation and apoptosis, is a complex area which as yet is very poorly described. However, it is generally believed that T-cells play a key role in the induction of apoptosis in epithelial cells and thus the potential to control tumour progression. Furthermore, a number of exocrine factors including inflammatory cytokines and growth factors such as interleukin 6 (IL-6) (6) and epidermal growth factor (EGF) (7) have also been demonstrated to influence cell proliferation. The proliferating colonocyte is exposed not only to systemic signalling, but also to influences from the luminal side, such as secondary bile acids, dietary carcinogens and bacterial metabolites, and possibly direct contact with bacteria via toll-like receptors (8). The importance of each of these factors in mucosal homeostasis and tumour initiation and promotion is as yet unclear, but it is recognized that dietary fatty acids can influence both the level of exposure to such exogenous factors and the sensitivity of the cell to associated signalling pathways.

Loss of control of tissue homeostasis occurs with age and is believed to be a result of a number of initiating factors. There is a purely statistically increased risk over time of spontaneous mutations escaping repair processes. This may be exacerbated by methylation of $\mathrm{CpG}$ islands in the promoter regions of proto-oncogenes such that they are switched off and no longer control key mechanisms underlying homeostasis, particularly DNA repair (9). DNA adducts and subsequent mutations as a result of mismatch repair may result from a number of potential toxins or as a result of oxidative stress, but the importance of this route is disputed as the mutation patterns seen in humans are not typical of such negative factors, apart perhaps from exposure to nitrosamines (10). However, it is well recognized that an accumulation of mutations in the DNA eventually leads to the development of tumours, with certain key genes (APC, K-ras, p53) being commonly found to be altered at different stages. To study how diet may prevent this process it is important to identify early markers of cancer risk. Increased cell proliferation and the expanded crypt zone are currently still the best recognized early markers of risk, although more recent evidence suggests that reduced apoptosis provides a better surrogate, in relation to the development of both adenoma in humans (11) and tumour in rats (12).

\section{Observational studies in relation to fatty acid intake}

Historically, high intakes of dietary fat have been linked to an increased risk of a number of cancers, including colorectal cancer, but the evidence has been inconsistent (13). In recent years the focus of research has centred more on the type of fat consumed and cancer risk, but even here the evidence from epidemiological studies is not convincing. However, animal studies do provide convincing evidence that certain fatty acids, in particular the very long-chain n-3 polyunsaturated fatty acids (PUFAs) found in oil-rich fish, may be protective. Probably the most consistently reported factors associated with increased risk of colorectal cancer are obesity and lack of exercise (14). The mechanisms underlying obesity-driven increased cancer risk are not known, but may relate to the chronic inflammatory state associated with metabolic syndrome. It is therefore conceivable that $n-3$ fatty acids would positively influence this by downregulating systemic or local inflammation. In contrast, diets with a high total fatty acid component are associated with high total calorie intake and obesity.

Of four well-conducted case-control studies, only one has shown significant protection associated with n-3 fatty acid consumption. In a recent systematic review MacLean et al. collated data 
from nine studies involving seven different cohorts in which colorectal cancer risk and n-3 fatty acid intake were assessed, and concluded that there was no significant association between n-3 fatty acid intake and disease risk (15). They also reported on fish intake and colorectal cancer, and again found no evidence to support any benefit of a high intake of fish. However, this analysis did not include the data from the European Prospective Investigation into Cancer and Nutrition (EPIC) on fish consumption and colorectal cancer risk published in the previous year (16), which reported a highly significant negative association between fish consumption and disease in those consuming more than $40 \mathrm{~g}$ of fish per day. This last study has the benefit not only of large size, but also of a wide range of fish consumption in the population studied, while in the majority of previous studies the highest percentile of fish consumption included intakes as low as one portion a week $(\sim 20 \mathrm{~g}$ per day). Whether the potential benefits of fish consumption are explicable in terms of their unusual fatty acid profiles is not clear from these data. This question is currently being addressed by a human intervention trial funded as part of the European Union integrated project SEAFOODplus (http:// www.seafoodplus.org/).

It should also be remembered that the quality of data relating to $\mathrm{n}-3$ fatty acid intake, collected using food frequency questionnaires, is often very poor, as is the fatty acid compositional data in food tables on which estimates of intake are based. In addition, few studies have been able to distinguish adequately between marine and plant sources of n-3 fatty acids, which are predicted to have very different biological activities, and only some studies include the consumption of fish oil supplements. Furthermore, animal studies would suggest that it is the ratio of n-3 to n-6 fatty acids that may be important in relation to risk (17), yet this analysis is rarely reported.

The impact of nutrient-gene interactions has recently been highlighted in an epidemiological study on fish consumption and single nucleotide polymorphisms (SNPs) for genes related to eicosanoid metabolism (18). For example, fish consumption is shown to be associated with reduced colorectal cancer risk in individuals with the most common CC genotype of the c.-789C-T SNP in peroxisome proliferator activated receptor- $\delta$ (PPAR- $\delta$ ), but in those with the CT or TT polymorphisms the reverse appears to be the case. Similarly, protection by fish consumption is particularly associated with those with the AG/GG forms of the Cox-2 SNP c.1329A $\rightarrow \mathrm{G}$ who otherwise have an elevated risk; therefore, epidemiological evidence should take into account the impact of a number of SNPs.

\section{Intervention studies in relation to fatty acid intake}

There are no long-term n-3 fatty acids intervention studies with cancer as the main endpoint, and although nine report on cancer risk even the largest of these trials, the GISSI-Prevenzione trial, only reports total cancer incidence (19). The prolonged period of development in relation to colorectal cancer risk makes it extremely difficult to undertake human intervention trials with tumour as an endpoint. For this reason, intermediate disease markers such as aberrant crypt foci and polyps/adenomas have been used in a number of studies, as have markers more related to mechanisms of development, such as cell proliferation, apoptosis and DNA damage and most recently methylation of $\mathrm{CpG}$ islands in the promoter regions of proto-oncogenes (20). These methods all require examination of the colon and usually collection of biopsy samples and are therefore not straightforward to implement in the context of large-scale intervention trials. However, human intervention trials have shown a protective effect of $n-3$ supplementation on crypt cell proliferation $(21,22)$ and apoptosis $(23)$. These results in humans are replicated in a diverse range of animal models using chemical carcinogens: dimethylhydrazine (DMH) and azoxymethane (12, 24), the APC ${ }^{\text {min }}$ mouse model (25) and implantation of tumour cells (26). Such studies also provide evidence for the relative protective effects of different fatty acids, such that eicosapentaenoic acid (EPA) and docosahexaenic acid (DHA) provide most protection, compared with a sunflower or safflower oil, or even an olive oil diet. The data on $\alpha$-linolenic acid and linseed oil are inconsistent, with some studies suggesting a small protective effect on tumour development and others suggesting an increased risk relative to sunflower oil, although not to the extent that conjugated linoleic acid (27). In the rat DMH model, fish oil (n-3 diet) suppresses cell proliferation and increased apoptosis relative to corn oil-fed (n-6) animals when fed after exposure to the carcinogen (28). This suggests a 
post-initiation protective effect of $\mathrm{n}-3$ consumption that is maintained for up to 18 weeks (12) when a reduction in aberrant crypt factor has been reported. These observations are consistent with epidemiological data provided by $\mathrm{Oh}$ et al. (29) showing that there maybe a post-initiation protective effect of fish consumption.

\section{Mechanisms underlying the effects of fatty acids on colorectal cancer risk}

The mechanisms by which n-3 fatty acids reduce cell proliferation and increase apoptosis are not well understood and are likely to be multifaceted. Redox modification would appear to be an important aspect in relation to induction of apoptosis. Rats given L-buthionine-[S,R]-sulfoximine (BSO) in their drinking water, to reduce intracellular glutathione levels, have increased levels of apoptosis, an effect that is exacerbated by the replacement of $n-6$ fatty acids in the diet with n-3 fatty acids (30). In cell culture, pretreatment with lipid-soluble antioxidants prevents apoptosis and loss of mitochondrial membrane potential (31), supporting the hypothesis that the oxidizability of long-chain, highly unsaturated PUFAs is important in relation to their protective role. Classically, induction of apoptosis has been considered to be regulated post-transcriptionally with procaspases always ready to be activated in response to either intrinsic or extrinsic signalling. However, it is entirely feasible that the sensitivity of a cell to a proapoptotic signal may be modified by altering not only the structure of cellular membranes, including mitochondrial membranes, but also gene expression of proteases and receptors involved in apoptosis. Potential mechanisms relating to dietary fatty acids include modification of Fas-L expression and/or activity on the cell surface, changes in mitochondrial potential, down-regulation of antiapoptotic molecules such as bcl-2 and up-regulation of proapoptotic molecules (23). Control of gene expression in relation to apoptosis, cell proliferation and differentiation by fatty acids may be exerted through binding to PPAR receptors. Both PPAR $-\gamma$ and PPAR $-\delta$ are expressed in the colon, are rarely mutated during tumour progression, and control genes associated with cell proliferation, apoptosis and differentiation (32). PPAR- $\gamma$ activation has been shown to induce apoptosis and reduce adenoma number in the colon, possibly by down-regulating Cox-2 expression (33). However, in the APC ${ }^{\text {min }}$ mouse some synthetic ligands have been reported to increase tumour progression, but others are protective (34). As natural ligands, n-3 fatty acids are protective in this model (25), yet n-3 and n-6 fatty acids show similar affinity for PPAR- $\gamma$. However, n-3 fatty acids have a higher affinity for the heterodimer retinoid $\mathrm{X}$ receptor- $\alpha(\mathrm{R} \times \mathrm{R}-\alpha)$ (35), so differential effects may be explicable in these terms. Alternatively, oxidized lipids increase PPAR- $\gamma$-controlled luciferase expression more than the unoxidized forms (36). The long-chain PUFAs found in fish oil, which oxidize more readily than, for example, linolenic acid, may therefore bind more strongly to PPARs and have an exacerbated effect. Normally, PPAR $-\gamma$ is increasingly expressed as cells move up the crypt axis and onto the luminal surface, but in some tumours and in the APC ${ }^{\text {min }}$ mouse PPAR $-\gamma$ is overexpressed as a result of $\beta$-catenin overexpression (37). The APC protein is also reported to control PPAR- $\delta$ expression through the $\beta$-catenin-Tcf- 4 pathway, such that wild-type APC promotes degradation of $\beta$-catenin and downregulation of PPAR- $\delta$ (38) and, although luciferase reporter assays suggest that PUFAs are agonists for PPAR- $\gamma / \mathrm{R} x \mathrm{R}-\alpha$ in the colon (35), research based on gel shift assays suggests that DHA, and to a lesser extent EPA, inhibits PPAR- $\delta$ and PPAR $-\gamma$ binding to the response element (39). The target genes for PPAR- $\delta$ are those associated with increased cell proliferation, such as c-myc and cyclin D1, and suppression of apoptosis. These results suggest that PUFAs may act by both reducing the proliferative effects of PPAR $-\delta$ overexpression and increasing the proapoptotic effects of PPAR- $\gamma$, but interpretation of the literature remains equivocal.

A diverse range of alternative mechanisms to explain the protective effect of $n-3$ fatty acids in relation to colorectal cancer has been proposed and comprehensively reviewed in two recent articles (40, 41). These include a number of mechanisms involving extrinsic influences on the colonocyte genotype and phenotype from both the colonic lumen and the circulation, as well as local intercellular signalling molecules including nitric oxide. For example, in the lumen n-3 fatty acids may change the bacterial conversion of bile acids to less genotoxic secondary bile acids and reduce the production polyamines. The hyperproliferative effects of circulating levels of insulin-like growth factor-II (IGF-II) are counteracted by the ability of n-3 PUFAs to induce the IGF binding protein IGFBP-6. Those of EGF are by chronic exposure to lipid peroxides, which 
down-regulate ornithine decarboxylase activity. It has also been demonstrated using reporter assays that DHA down-regulates EGF-mediated AP-1 (jun-fos) transcription factor-controlled gene expression, which would be predicted to reduce cell transformation and tumour promotion (42). Colonic lymphocytes from mice fed fish oil rather than corn oil have a lower proliferative response to a number of proinflammatory cytokines (43). The n-3 fatty acids are well recognized for their antiinflammatory properties, particularly EPA, which acts as a substrate for the cyclooxygenase and lipoxygenase-catalysed formation of eicosanoids and related oxidized metabolites, competing with arachidonic acid and generally forming less inflammatory mediators. As inflammation may be important in relation to increased tumour risk, such differences are predicted to be beneficial. Many studies have shown reduced prostaglandin-2 (PGE2) production in response to consumption of $n-3$ fatty acids in both colorectal tumour cell lines and tissue. In the latter case, it is uncertain whether the effect relates to PGE formation by the epithelial cells or that of associated leukocytes. However, there is considerable support for the concept that PGE-2 is a tumour promoter, as treatment with Cox-2 inhibitors reduces tumour number in several systems (44). Furthermore, there is evidence to suggest that arachidonic acid-derived eicosanoids are involved in tumour promotion via PPARs (38) and, for example, 15d-PGJ2 can inhibit PPAR- $\gamma$-induced cell death (45).

Overall, the evidence based on a combination of observational and mechanistic studies would suggest that n-3 fatty acids may well play an important role in the prevention of the development of colorectal cancer.

\section{References}

1. Parkin DM, Whelan S, Ferlay J, Storm H. Cancer incidence in five continents. Vols I-VIII. IARC CancerBase No 7. Lyon: IARC; 2005.

2. Key TJ, Allen NE, Spencer EA, Travis RC. The effect of diet on risk of cancer. Lancet 2002; 360: 861-8.

3. Johnson IT. New approaches to the role of diet in the prevention of cancers of the alimentary tract. Mutat Res 2004; 551: 9-28.

4. Wong WM, Wright NA. Cell proliferation in gastrointestinal mucosa. J Clin Pathol 1999; 52: 321-33.

5. Martin MS, Hammann A, Martin F. Gut-associated lymphoid tissue and 1,2-dimethylhydrazine intestinal tumors in the rat: an histological and immunoenzymatic study. Int J Cancer 1986; 38: 75-80.

6. Becker C, Fantini MC, Wirtz S, Nikolaev A, Lehr HA, Galle PR, et al. IL-6 signalling promotes tumor growth in colorectal cancer. Cell Cycle 2005; 4: 217-20.

7. Eling TE, Glasgow WC. Cellular proliferation and lipid metabolism: importance of lipoxygenases in modulating epidermal growth factor-dependent mitogenesis. Cancer Metastasis Rev 1994; 13: 397-410.

8. Singh JC, Cruickshank SM, Newton DJ, Wakenshaw L, Graham A, Lan J, et al. Toll-like receptor-mediated responses of primary intestinal epithelial cells during the development of colitis. Am J Physiol Gastrointest Liver Physiol 2005; 288: G514-24.

9. Belshaw NJ, Elliott GO, Williams EA, Bradburn DM, Mills SJ, Mathers JC, et al. Use of DNA from human stools to detect aberrant $\mathrm{CpG}$ island methylation of genes implicated in colorectal cancer. Cancer Epidemiol Biomarkers Prev 2004; 13: 1495-501.

10. Bingham SA, Hughes R, Cross AJ. Effect of white versus red meat on endogenous $\mathrm{N}$-nitrosation in the human colon and further evidence of a dose response. $\mathbf{J}$ Nutr 2002; 132: 3522-5S.

11. Martin C, Connelly A, Keku TO, Mountcastle SB, Galanko J, Woosley JT, et al. Nonsteroidal anti-inflammatory drugs, apoptosis, and colorectal adenomas. Gastroenterology 2002; 123: 1770-7.

12. Chang WC, Chapkin RS, Lupton JR. Predictive value of proliferation, differentiation and apoptosis as intermediate markers for colon tumorigenesis. Carcinogenesis 1997; 18: 721-30.

13. WCRF. Food, nutrition and the prevention of cancer: a global perspective. In: Potter J. ed. London: World Cancer Research Fund; 1997.

14. Frezza EE, Wachtel MS, Chiriva-Internati M. Influence of obesity on the risk of developing colon cancer. Gut 2006; 55: 285-91.

15. MacLean $\mathrm{CH}$, Newberry SJ, Mojica WA, Khanna $\mathrm{P}$, Issa AM, Suttorp MJ, et al. Effects of omega-3 fatty acids on cancer risk: a systematic review. JAMA 2006; 295: 403-15.

16. Norat T, Bingham S, Ferrari P, Slimani N, Jenab M, Mazuir M, et al. Meat, fish, and colorectal cancer risk: the European Prospective Investigation into cancer and nutrition. J Natl Cancer Inst 2005; 97: 906-16.

17. Bartram HP, Gostner A, Reddy BS, Rao CV, Scheppach W, Dusel G, et al. Missing anti-proliferative effect of fish oil on rectal epithelium in healthy volunteers consuming a high-fat diet: potential role of the n-3:n-6 fatty acid ratio. Eur J Cancer Prev 1995; 4: 231-7.

18. Siezen CL, Tijhuis MJ, Kram NR, van Soest EM, de Jong DJ, Fodde R, et al. Protective effect of nonsteroidal anti-inflammatory drugs on colorectal adenomas is modified by a polymorphism in peroxisome proliferator-activated receptor delta. Pharmacogenet Genomics 2006; 16: 43-50.

19. Dietary supplementation with n-3 polyunsaturated fatty acids and vitamin $\mathrm{E}$ after myocardial infarction: results of the GISSI-Prevenzione trial. Gruppo Italiano per lo 
Studio della Sopravvivenza nell'Infarto miocardico. Lancet 1999; 354: 447-55.

20. Issa JP, Ahuja N, Toyota M, Bronner MP, Brentnall TA. Accelerated age-related $\mathrm{CpG}$ island methylation in ulcerative colitis. Cancer Res 2001; 61: 3573-7.

21. Anti M, Marra G, Armelao F, Bartoli GM, Ficarelli R, Percesepe A, et al. Effect of omega-3 fatty acids on rectal mucosal cell proliferation in subjects at risk for colon cancer. Gastroenterology 1992; 103: 883-91.

22. Bartoli GM, Palozza P, Marra G, Armelao F, Franceschelli P, Luberto C, et al. n-3 PUFA and alpha-tocopherol control of tumor cell proliferation. Mol Aspects Med 1993; 14(3): 247-52.

23. Cheng J, Ogawa K, Kuriki K, Yokoyama Y, Kamiya T, Seno K, et al. Increased intake of n-3 polyunsaturated fatty acids elevates the level of apoptosis in the normal sigmoid colon of patients polypectomized for adenomas/ tumors. Cancer Lett 2003; 193: 17-24.

24. Reddy BS, Sugie S. Effect of different levels of omega-3 and omega-6 fatty acids on azoxymethane-induced colon carcinogenesis in F344 rats. Cancer Res 1988; 48: 6642-7.

25. Paulsen JE, Elvsaas IK, Steffensen IL, Alexander J. A fish oil derived concentrate enriched in eicosapentaenoic and docosahexaenoic acid as ethyl ester suppresses the formation and growth of intestinal polyps in the Min mouse. Carcinogenesis 1997; 18: 1905-10.

26. Calder PC, Davis J, Yaqoob P, Pala H, Thies F, Newsholme EA. Dietary fish oil suppresses human colon tumour growth in athymic mice. Clin Sci (Colch) 1998; 94: 303-11.

27. Wahle KW, Heys SD, Rotondo D. Conjugated linoleic acids: are they beneficial or detrimental to health? Prog Lipid Res 2004; 43: 553-87.

28. Latham P, Lund EK, Johnson IT. Dietary n-3 PUFA increases the apoptotic response to 1,2-dimethylhydrazine, reduces mitosis and suppresses the induction of carcinogenesis in the rat colon. Carcinogenesis 1999; 20: 645-50.

29. Oh K, Willett WC, Fuchs CS, Giovannucci E. Dietary marine n-3 fatty acids in relation to risk of distal colorectal adenoma in women. Cancer Epidemiol Biomarkers Prev 2005; 14: 835-41.

30. Latham P, Lund EK, Brown JC, Johnson IT. Effects of cellular redox balance on induction of apoptosis by eicosapentaenoic acid in HT29 colorectal adenocarcinoma cells and rat colon in vivo. Gut 2001; 49: 97-105.

31. Ng Y, Barhoumi R, Tjalkens RB, Fan YY, Kolar S, Wang $\mathrm{N}$, et al. The role of docosahexaenoic acid in mediating mitochondrial membrane lipid oxidation and apoptosis in colonocytes. Carcinogenesis 2005; 26: 1914-21.

32. Murtaugh MA, Ma KN, Caan BJ, Sweeney C, Wolff R, Samowitz WS, et al. Interactions of peroxisome proliferator-activated receptor $\gamma$ and diet in etiology of colorectal cancer. Cancer Epidemiol Biomarkers Prev 2005; 14: 1224-9.

33. Yang WL, Frucht H. Activation of the PPAR pathway induces apoptosis and COX-2 inhibition in HT-29 human colon cancer cells. Carcinogenesis 2001; 22: 1379-83.

34. Niho N, Takahashi M, Kitamura T, Shoji Y, Itoh M, Noda T, et al. Concomitant suppression of hyperlipidemia and intestinal polyp formation in Apc-deficient mice by peroxisome proliferator-activated receptor ligands. Cancer Res 2003; 63: 6090-5.

35. Fan YY, Spencer TE, Wang N, Moyer MP, Chapkin RS. Chemopreventive $n-3$ fatty acids activate $\mathrm{RXR} \alpha$ in colonocytes. Carcinogenesis 2003; 24: 1541-8.

36. Bull AW, Steffensen KR, Leers J, Rafter JJ. Activation of PPAR gamma in colon tumor cell lines by oxidized metabolites of linoleic acid, endogenous ligands for PPAR gamma. Carcinogenesis 2003; 24: 1717-22.

37. Jansson EA, Are A, Greicius G, Kuo IC, Kelly D, Arulampalam V, et al. The Wnt/beta-catenin signaling pathway targets PPAR $\gamma$ activity in colon cancer cells. Proc Natl Acad Sci USA 2005; 102: 1460-5.

38. Gupta RA, Tan J, Krause WF, Geraci MW, Willson TM, Dey SK, et al. Prostacyclin-mediated activation of peroxisome proliferator-activated receptor delta in colorectal cancer. Proc Natl Acad Sci USA 2000; 97: 13275-80.

39. Lee JY, Hwang DH. Docosahexaenoic acid suppresses the activity of peroxisome proliferator-activated receptors in a colon tumor cell line. Biochem Biophys Res Commun 2002; 298: 667-74.

40. Roynette CE, Calder PC, Dupertuis YM, Pichard C. n-3 polyunsaturated fatty acids and colon cancer prevention. Clin Nutr 2004; 23: 139-51.

41. Larsson SC, Kumlin M, Ingelman-Sundberg M, Wolk A. Dietary long-chain n-3 fatty acids for the prevention of cancer: a review of potential mechanisms. Am J Clin Nutr 2004; 79: 935-45.

42. Liu G, Bibus DM, Bode AM, Ma WY, Holman RT, Dong Z. Omega 3 but not omega 6 fatty acids inhibit AP-1 activity and cell transformation in JB6 cells. Proc Natl Acad Sci USA 2001; 98: 7510-5.

43. Kuratko CN. Proliferation of colonic lymphocytes in response to inflammatory cytokines is lower in mice fed fish oil than in mice fed corn oil. Cancer Lett 2000; 148: 27-32.

44. Tapiero H, Ba GN, Couvreur P, Tew KD. Polyunsaturated fatty acids (PUFA) and eicosanoids in human health and pathologies. Biomed Pharmacother 2002; 56: 215-22.

45. Shimada T, Kojima K, Yoshiura K, Hiraishi H, Terano A. Characteristics of the peroxisome proliferator activated receptor gamma (PPAR $\gamma$ ) ligand induced apoptosis in colon cancer cells. Gut 2002; 50: 658-64.

\footnotetext{
Elizabeth Lund

Institute of Food Research

Norwich Research Park

Colney

Norwich NR4 7UA

UK

Tel: +44 I603255347

E-mail: liz.lund@bbsrc.ac.uk
} 